\title{
EDITORIAL
}

\section{Prediction of the course and outcome of depression ${ }^{1}$}

Whether defined as a symptom, a syndrome, a single disease, or a 'spectrum' of biopsychosocial manifestations, depression is at present the most common mental disorder in all the urbanizedindustrial societies and its frequency appears to be increasing in the developing countries. Klerman's observation that, after an 'age of anxiety', the world is entering an 'age of melancholy' (Klerman, 1978) has been remarkably borne out by recent epidemiological data which demonstrate trends of increasing incidence and prevalence of depressive illnesses.

\section{EPIDEMIOLOGICAL TRENDS}

Data from earlier community surveys and other population-based studies refer mainly to the prevalence and incidence of severe affective psychoses (i.e. the spectrum of 'neurotic' depressive illness was not included). The point prevalence figures of the 1930s were within the range $0.3-2.7$ per 1000 population (Brugger, 1933; Strömgren, 1938). Using a somewhat broader concept, figures as high as $13 \cdot 1$ per 1000 have been reported from population censuses in the 1970s (Nielsen, 1976). More recently, the data from the Epidemiological Catchment Areas (ECA) studies in the USA showed a 6-month prevalence for all the DSM-III affective disorders of between 46 and 65 per 1000 . The prevalence of major depressive episodes was 22-35 per 1000 (Myers et al. 1984).

The annual incidence of first episodes of affective psychoses is of the order of $0.4-2.7$ per 1000 population at risk (Nielsen, 1976; L. Helgason, 1977). The index of morbid risk (expectancy) was estimated to be $0 \cdot 31-0.42 \%$ in the 1930 s (Dahlberg \& Stenberg, 1931 ; Brugger, 1933) and $1 \cdot 20-3 \cdot 23 \%$ in the 1960s and 1970s (T. Helgason, 1964; L. Helgason, 1977). The index of lifetime prevalence used in the recent ECA studies had much higher values: $6 \cdot 1-9 \cdot 5 \%$ for any affective disorder and $3 \cdot 7-6 \cdot 7 \%$ for DSM-III major affective episodes (Robins et al. 1984).

The lack of diagnostic concordance between studies, the incomparability of samples, and other methodological confounding factors may raise doubts about the validity of any interpretation of such large differences in the results produced by different groups of investigators at different points in time. However, at least three recent studies seem to indicate that real changes may have occurred in the incidence of depressive disorders over the past 2-3 decades.

The first piece of evidence comes from Hagnell's extended follow-up of a population originally surveyed by Essen-Möller et al. (1956) in the Lundby study in southern Sweden. On the basis of a 25-year follow-up, Hagnell et al. (1982) calculated age- and sex-standardized incidence rates for each 5 -year period, as well as cumulative probabilities (morbid risk) of developing a depressive illness associated with mild, moderate or severe impairment up to the age of 80 . The data indicated a tenfold increase of the risk of depression with a severe and medium impairment for males in the age group 20-39 in the period 1957-72 compared with the period 1947-57.

The second piece of evidence is provided by Weissman (1985) on the basis of data collected in the ECA studies in the USA. In replicating earlier findings reported by Klerman (1978), she demonstrated a marked birth cohort effect on the cumulative incidence rates of major depression, with a highly significant shift towards higher rates and earlier onset in females born after 1935.

The third piece of evidence is contained in a British study covering a 20 -year period of a single general practice in London (Dunn \& Skuse, 1981). Using a log-linear model, it was shown that both

\footnotetext{
1 Based on a paper presented at a European Medical Research Council workshop ' Prediction of Course and Outcome in Depressive Illness: Needed Areas for Research', held in Cork, Ireland, 28-31 August 1985. The remaining papers of the workshop are to be published as a supplement to Acta Psychiatrica Scandinavica. Address for correspondence: Dr A. Jablensky, 15 Dim. Nestorov Str., Sofia 1431, Bulgaria.
} 
the prevalence and incidence of depression in males were rising in the period 1957-76 (in females, there was an increase in prevalence, but not in incidence).

These epidemiological trends show a sex difference between North America and Europe, but both point in the same direction. The underlying factors remain totally unknown, although Hagnell and Weissman have considered environmental explanations in terms of lifestyle and social change. Whatever the ultimate explanation, these data require replication in other populations. If confirmed, they will strengthen the prediction (Sartorius, 1978) of an increasing frequency of depression worldwide, due to the combined effects of demographic, social and biological causes.

\section{NEED FOR A REFINEMENT IN THE PROGNOSTIC EVALUATION OF AFFECTIVE DISORDERS}

If the epidemiological trends represent an intriguing problem to the research worker and a concern for the public health planner, the expanded range of treatment modalities available in the 1980s poses special questions to the clinician in the management of the individual patient. In addition to new classes of antidepressant drugs, there are now new non-pharmacological methods (e.g. sleep deprivation, light stimulation) and psychotherapeutic techniques (cognitive therapy) which await evaluation and assessment of indications. Their potential is unlikely to be fully understood and utilized unless clinical trials take into account the various 'natural' patterns of course and outcome of the depressive disorders and their predictors.

The lack of an agreed common set of concepts and methods in diagnosing depression and describing its course continues to be an obstacle to improvements in our ability to formulate the prognosis of depressive illnesses. The 'contemporary confusion' (Kendell, 1976) affecting the diagnosis and classification of depressive disorders has not been resolved; if anything, it may even have become exacerbated. There is still a plethora of diagnostic 'systems' and ad hoc provisions for classifying affective disorders, which employ concepts and terms that are far from being equivalent or synonymous. Much of the clinical data collected in the past and used today for follow-up analysis is recorded in obsolete diagnostic terms that no longer correspond to the current usage. Although overall the differences between ICD-8 and ICD-9 are relatively minor, the former contains only 10 , while the latter includes 19 four-digit categories in which depression or mania is a major or significant feature (WHO, 1978). The so-called operational diagnostic criteria, including the CATEGO system for classifying clinical information (Wing et al. 1974), the St Louis criteria (Feighner et al. 1972), the Research Diagnostic Criteria (Spitzer et al. 1977) and DSM-III (APA, 1980), aim to reduce the subjective element in diagnostic decisions. However, these different criteria have been shown to identify only partially overlapping clinical populations, and the concordance between them is of a low order (Kendell \& Brockington, 1980). In spite of the increasing pressures on investigators to use operational criteria, many research workers prefer diagnostic concepts stemming from the particular tradition and setting within which they are operating.

The agreement on the criteria and methods used to describe course and outcome is equally poor. While in some instances such criteria are not specified at all, in other instances, where specific definitions are given for episode, interval, pattern of course, social functioning, etc., the criteria vary from study to study, making direct comparisons of results uncertain.

The removal or reduction of such obstacles should be seen as an important priority, and the World Health Organization, which has a mandate for establishing international standards for biomedical research, has a special role to play in this respect. Notwithstanding all these methodological reservations, a provisional summing up of current knowledge about the prognosis of affective disorders may help at this point, even if it could not aim to be comprehensive or conclusive.

The issue of prognosis in the depressive disorders can be approached in two ways. On one hand, available knowledge can be examined with a view to identifying a longitudinal profile or a 'natural history' of the group of depressive disorders. By aggregating information from different studies one could obtain actuarial estimates of basic parameters such as age of onset, sex ratio, different patterns of course, social outcome, and associated mortality. 
On the other hand, research data can be reviewed with the aim of obtaining more specific predictions to supplement the actuarial estimates and thus refine the prognosis in the individual case.

\section{THE ‘NATURAL HISTORY’ OF DEPRESSIVE DISORDERS}

First episodes of depressive illness may occur at any age. In contrast to schizophrenia, where disease onsets show a characteristic clustering in the age group 15-24, the onsets of depressive illnesses are spread over the entire life span in both sexes, as demonstrated clearly in the 25-year prospective Lundby study (Hagnell et al. 1982). This general pattern is likely to be sustained even if the recently reported trends of increasing incidence in younger males in Europe, or younger females in the USA continue. However, if depressive illnesses are split into bipolar (manic-depressive) and unipolar (recurrent depressive) subgroups, the median age of onset would shift towards younger age for the bipolar patients but remain within a less clearly determined range for the unipolar patients (Angst, 1980).

The sex ratio almost invariably shows a preponderance of females $(F: M$ is of the order $2-3: 1)$, but there are some indications that the gap between the incidence rates in the two sexes may be diminishing.

Recent epidemiological research focusing on possible psychosocial contributions to the aetiology of depression (Brown \& Harris, 1978; Brown \& Prudo, 1981) has shown that a provoking agent could be identified in $80-89 \%$ of all the episodes of depression in non-hospitalized community samples; in $65-73 \%$ of the cases this agent was considered 'severe'. These findings are not entirely conclusive, since most of the studies have included women only and the classification of the clinical findings employed is not directly comparable with the data obtained in other settings. However, seen in conjunction with the results of other studies, they point to an almost ubiquitous, though probably non-specific, role of stressful life events in the precipitation of depressive illnesses and reinforce the doubts about the validity of a categorical distinction between 'endogenous' and 'reactive' affective disorders.

The typical course of depressive disorders is episodic, and recent research findings have not altered significantly the 'stereotype' of mood disorders established since Kraepelin (1921). However, there is undoubtedly a proportion of cases characterized by a single episode of depression remitting without relapse. The ascertainment of such cases probably depends on the length of follow-up. Coryell \& Winokur (1982) reviewed earlier studies and concluded that this proportion tends to increase (rather than decrease) with the length of follow-up. They also pointed out that affective episodes tend to occur 'in bursts' followed by long remissions and that at no point in the course of an affective disorder could future relapse be predicted with any certainty from the number of past episodes.

Data from the WHO International Pilot Study of Schizophrenia (IPSS) (WHO, 1979) which, in addition to the schizophrenic cases included a series of patients with psychotic depressions, indicate that 2 years after the index episode $35 \%$ of the depressive patients were in a complete remission without further episodes and that another $21 \%$ had some residual symptoms but no relapses. Only $7 \%$ of the depressive patients continued to be severely ill, without remission from the index episode, while another $37 \%$ had had one or more relapses. Unpublished data from the 5-year follow-up of another multi-centre WHO study on depression (Sartorius et al. 1983; Jablensky et al. 1981) show similarly high percentages of single-episode course (49\% in Basle, $31 \%$ in Montreal, and $52 \%$ in Tokyo and Nagasaki) in patients meeting specified criteria for severity of the index depressive episode. Chronicity, i.e. unremitting index episode over the 5 years of the follow-up, was observed in $18 \%$ of the Basle cases, $3 \%$ of the Montreal cases, and $7 \%$ and $13 \%$ respectively of the Tokyo and Nagasaki cases. Varying proportions of patients in the different research centres had subsequent episodes, but fewer than $10 \%$ in any centre had more than 2 relapses during the 5 years of the follow-up.

The question whether bipolar and unipolar illnesses exhibit consistent differences in their long-term course has not been answered satisfactorily. The 40-year follow-up of a cohort of patients 
by Winokur (1975) and by Tsuang (1980) failed to demonstrate such differences. In the WHO IPSS $39 \%$ of the patients who were in a manic psychotic episode at the point of inclusion (and therefore could be classified as bipolar cases) were in remission at the 2-year follow-up and had had no relapses; $2 \%$ continued to be in the index psychotic episode; and $58 \%$ had had one or more relapses. Although the course of the IPSS manic patients tended to be less favourable compared with the IPSS depressives, the relatively short follow-up period and the modest size of the patient series (71 patients with psychotic depression and 53 patients with mania) preclude firm conclusions.

In bipolar patients with many recurrences, Angst (1980) observed an initial trend of diminishing length of the intervals between episodes, with subsequent stabilization. Patients with early onset (before age 40) and patients with late onset (after 40) had the same average frequency and length of episodes. In unipolar depressions, however, episodes in late onset cases tended on average to be longer than episodes in early onset patients, although the frequency of the episodes did not differ between the two groups (Angst, 1979).

The proportion of the follow-up period during which patients exhibit marked depressive or manic symptoms (i.e. the sum of the duration of all psychotic episodes) was also calculated in the IPSS series of affective disorders. The majority of patients with an index episode of depression $(54 \%)$ spent less than $(15 \%)$ of the 2-year period in such episodes; only $9 \%$ of these patients were psychotic for $76-100 \%$ of the period. Virtually the same proportions were observed in the series of cases with an index episode of mania.

Affective symptomatology tends to 'breed true', in the sense that few patients develop illnesses of a qualitatively different kind (e.g. schizophrenic) after an initial typical affective illness (Paykel et al. 1976). While the occurrence of affective episodes or of sequences of depressive and manic illnesses is not an uncommon phenomenon after a first episode of schizophrenia (Sheldrick et al. 1977), secondary development of schizophrenia is rare after an initial affective illness (with the possible exception of atypical episodes of 'emotional turmoil' in adolescence). In the second WHO study referred to above, only 3 out of 155 depressive patients followed up for 5 years developed schizophrenic or paranoid psychotic episodes after the index depressive episode.

The social outcome of affective disorders, in terms of occupational adjustment, functioning in the family and the community, etc. is correlated with the long-term pattern of course. The WHO 2-year data did not reveal any differences in the social outcome of the patients with index depression and the patients with index mania (severe social impairment was observed in less than $10 \%$ of the cases in either group). However, Tsuang's 40-year follow-up data indicated that a higher proportion of the cases with an index manic illness, compared with the index depressives, fell into the poor occupational and marital adjustment categories, other factors being equal (Tsuang, 1980).

Excessive mortality, mainly due to suicide, is a well known risk in affective disorders. However, hospital-based statistics derived from the Norwegian national psychiatric case register (Saugstad \& Ødegaard, 1979) indicate a long-term downward trend for the age-adjusted death rates per 10000 for affective psychoses between 1950 and 1974 (249,274 and 179 for males, and 189, 230 and 155 for females, for the periods 1950-62, 1963-8 and 1969-74 respectively). In the same period the age-adjusted death rates for patients with a diagnosis of schizophrenia have increased in males, and shown some decrease in females. In both sexes, however, the mortality of schizophrenics in the 1970s has exceeded that of affective patients, which is in marked contrast to the earlier pattern. In the Iowa 500 study (Tsuang \& Woolson, 1978; Tsuang, 1978; Tsuang et al. 1980), excess mortality was observed in both schizophrenics and affective patients. Among the depressive and manic patients this excess was limited to the first decade of the follow-up, while in the schizophrenics it was present throughout the follow-up. Survival curves indicated that survival time was significantly shortened for both male and female depressives, and for female manics (but not for male manics). Suicide and accidental death accounted for an excess proportion of all deaths $(11.1 \%$ among manics and $9.3 \%$ among depressives, compared with $1.9 \%$ among the surgical control cases). An important finding in this study, which corroborates earlier data (e.g. Guze \& Robins, 1970), is that suicides tend to cluster in the early part of the follow-up period, e.g. in the first year after a discharge from treatment of an index episode, and that in the course of time the risk of suicide tends to diminish. 
This is not at all the case in schizophrenic patients among whom the risk of suicide remains constant over longer periods of time.

The modifiability of the short-term (6 months) course of depressive illnesses by ECT and imipramine was adequately documented for the first time in a controlled multi-centre study involving 250 patients in the early 1960s (MRC Clinical Psychiatry Committee, 1965). In spite of the large volume of research which has demonstrated the effects of different treatments on the course of individual episodes, the evidence concerning the impact of treatment on the long-term course of affective disorders is less conclusive, with the possible exception of studies showing a stabilizing effect of lithium in bipolar patients with a history of multiple relapses. More recently, two major studies (Glen et al.1984; Prien et al. 1984) have shown, under carefully controlled conditions, that the relapse rate over 3 and 2 years following recovery from a depressive episode is higher in patients receiving a placebo than among patients receiving (in the first study) lithium or amitriptyline, or (in the second study) imipramine, lithium, or a combination of the two.

The main features of studies providing evidence with prognostic implications for the course and outcome of depressive disorders are summarized in Table 1.

Table 1. Selected follow-up and prospective studies of depression with prognostic implications

\begin{tabular}{|c|c|c|c|c|}
\hline & $\begin{array}{l}\text { No. of } \\
\text { cases }\end{array}$ & $\begin{array}{l}\text { Length } \\
\text { of follow up } \\
\text { (years) }\end{array}$ & Diagnostic classification & Predictors \\
\hline Akiskal et al. (1978) & 100 & $3-4$ & Unipolar/bipolar & Clinical \\
\hline Akiskal et al. $(1983 a, b)$ & 206 & $1-9$ & Feighner & Clinical, genetic, pharmacological \\
\hline Angst et al. (1981) & 406 & $8-15$ & ICD-8 & Clinical \\
\hline Binder \& Angst (1981) & 600 & 4 & SCL-90R & Sociodemographic, self-ratings \\
\hline Bland \& Om (1982) & 108 & 15 & Fe1ghner & Clinical \\
\hline Brockington et al. (1982) & 125 & 6 & CATEGO/RDC/DSM-III & Diagnosis \\
\hline Copeland $(1983,1985)$ & 71 & 5 & $\begin{array}{l}\text { (a) Psychotic/neurotic } \\
\text { (b) Statistical clusters }\end{array}$ & Clinical \\
\hline Coryell \& Tsuang (1985) & $525(325)$ & 40 & DSM-III & Clinical \\
\hline Davidson \& Robertson (1985) & 82 & $1-32$ & Post-partum disorder & Clinical, sociodemographic \\
\hline Enzell (1984) & 589 & 3 & 'Depressive symptoms' & Clinical, social \\
\hline Keller et al. (1984) & 97 & 2 & RDC & Sociodemographic, clinical \\
\hline Prien et al. (1984) & 267 & 2 & RDC & Clincal, pharmacological \\
\hline Träskman et al. (1981) & 119 & $1-9$ & 'Depressed/non-depressed' & 5-HIAA, HVA, MHPG \\
\hline Videbech (1975) & 104 & & Manic-depressive & Clinical, genetic \\
\hline Winokur (1975) & $525(325)$ & 35 & Feighner & Sociodemographic, clinical \\
\hline WHO-IPSS (1979) & 123 & 2 & ICD-8/CATEGO & Sociodemographic, clinical \\
\hline
\end{tabular}

\section{SPECIFIC PREDICTORS OF COURSE AND OUTCOME}

The general description of the 'natural history' of affective disorders is not sufficient to determine the prognosis in the individual case. For this purpose, the 'generic' data need to be supplemented with information derived from studies focusing on more specific aspects of the course and outcome and their predictors.

Since one of the major distinctions within the group of affective disorders is that between bipolar and unipolar patterns, an important task in the individual case is the early and reliable assessment of the polarity of the illness. Most of the available data suggest that, in the absence of a past manic or hypomanic episode, the best predictors of a bipolar course are: (i) a family history of bipolar affective disorder; and (ii) onset at an early age (below 25). In addition, psychotic features, presence of retardation and hypersomnia, and a hypomanic response to antidepressant treatment within the first 4 weeks of its initiation augment the probability of a bipolar development. In females, depressive illnesses precipitated by childbirth more often assume a bipolar, rather than unipolar, course. Absence of specific predictors of bipolarity, especially in association with an introverted or 
anancastic premorbid personality, points to a probable non-bipolar course (single episode or recurrent depressions).

A number of studies have focused on tentative biological predictors of polarity in the affective disorders. For example, the urinary excretion of MHPG has been reported to be lower in bipolar patients than in unipolar patients (Schildkraut et al. 1978; Maas et al.1984), and the sensitivity of the dexamethasone suppression test (DST) has been found to be higher in detecting unipolar patients than in identifying bipolar cases (Feinberg \& Carroll, 1984). No single biochemical variable, however, has up to date been shown to discriminate reliably between unipolar and bipolar depressions and the clinical application of such tests is unwarranted, in spite of their research uses.

The establishment of the polarity of the illness does not facilitate the prediction of the length of an individual episode. In both bipolar and unipolar illnesses the duration of episodes varies greatly from subject to subject. It appears that for each individual the average duration of the affective episodes tends to be more or less constant; the length of future episodes can therefore be predicted from knowledge about the duration of past episodes. Both IPSS and other studies (e.g. Keller et al. 1984) have shown that a chronic course of depressive illness can be predicted if the episode has been in existence for a considerable length of time prior to the index assessment.

In the absence of bipolar features (which portend an increased risk of relapse) the prediction of a subsequent depressive episode following a remission of a depressive illness is extremely difficult.

Provoking factors like stressful life events are associated with an increased risk of relapse, especially in the presence of long-term vulnerablility factors such as lack of supportive social networks (Brown \& Prudo, 1981). Anhedonia and hyperreactivity to everyday stresses have been identified as early signs of an imminent relapse of depression (Quitkin et al. 1975).

While a large number of studies have focused on the prediction of the treatment response in the individual episode, few prospective investigations provide management and treatment guidelines with a view to the long-term course of affective disorders. An initial manic or mixed episode in a bipolar illness can predict a better response to lithium than to a maintenance antidepressant in the long run (Prien et al. 1984). Normal urinary secretion of norepinephrine associated with a low secretion of MHPG predicts good therapeutic response in bipolar patients, but not in unipolar cases; on the contrary, high levels of urinary metanephrine in association with lowered CSF 5-HIAA predict good treatment response in unipolar cases, but not in bipolars. High EMG activity in particular facial muscles (corrugator and zygomatic) has also been found to predict a good response to treatment in 'endogenous' depressives (Greden et al. 1984). Such laboratory investigations, however, are unlikely to be of help in the management of the large majority of depressive patients. In spite of the great number of clinical studies of antidepressants, the knowledge about clinical predictors of the effects of specific interventions on the long-term course of the disorder is unsatisfactory.

The base rate for the prediction of suicide risk in patients with affective disorders is generally high and a history of attempted suicide appears to predict both further attempts and completed suicide (Bürk \& Möller, 1985). A finding of considerable interest is that the CSF 5-HIAA is lowered in those affective patients who develop the syndrome of 'vital' depression and represent a subgroup at a particularly high risk of suicide (Träskman et al. 1981).

Few predictors of social outcome are specific to the affective disorders. It seems that the general prognostic factors related to social adjustment in most of the psychiatric (and non-psychiatric) disorders also provide support for the social prediction in affective disorders: premorbid personality, socio-economic status and education, and past occupational history. However, in contrast to the prediction of social outcome in schizophrenia, marital status does not appear to discriminate clearly between favourable and unfavourable course in affective disorders. 


\section{DIAGNOSTIC CLASSIFICATION AND THE PREDICTION OF COURSE AND OUTCOME}

A wide variety of classificatory principles and diagnostic criteria are being used in the assessment of affective disorders. Feighner's criteria (Feighner et al. 1972), the RDC (Spitzer et al. 1977) and DSM-III (APA, 1980) clearly predominate in North American research and are also applied in an increasing number of European studies. However, more traditional diagnostic concepts, such as the distinction between endogenous and reactive disorders, or between psychotic and neurotic affective illnesses, incorporated in ICD-8 and ICD-9, continue to be widely used.

Most of the major current 'systems' for classifying affective illnesses employ dichotomies, such as bipolar-unipolar, primary-secondary, endogenous-reactive, and so forth. Each of these concepts seems to have certain prognostic implications, but few of them have been sufficiently explored and validated. The association of the unipolar-bipolar distinction with the long-term course of the disorder and the relapse rate has already been mentioned. The primary-secondary dichotomy, as embodied in the RDC (Spitzer et al. 1977), also appears to be related to prognosis in so far as the course and outcome of a depression secondary to another psychiatric or non-psychiatric disorder would depend largely on the evolution of the underlying disease. The psychotic-neurotic and the endogenous-reactive distinctions, as long as they are interpreted in terms of severity and quality of symptomatology, and not aetiologically, predict with some accuracy the nature of symptoms in subsequent recurrences, but to a lesser degree (if at all) the risk of recurrences themselves. Similar considerations apply to the CATEGO classes for depressive disorders, D + (depressive psychoses), $\mathbf{R}$ (retarded depression), and $\mathbf{N}$ (neurotic depressions). While the distinction between $\mathrm{R}$ and $\mathrm{N}$ does not have any particular prognostic implication, the $\mathrm{D}+$ cases, in contrast to the group of $R$ and $\mathrm{N}$, have been shown to have a higher relapse rate, more chronicity and a tendency for psychotic symptoms to recur (Brockington et al. 1982).

\section{PRIORITIES FOR RESEARCH}

The overview of recent advances concerning the prediction of course and outcome in the affective disorders leads to the conclusion that the gains in knowledge have been modest. Amid a general quantitative and qualitative growth of scientific research into the aetiology and psychobiology of depression, and a wide recognition of the public health dimension of the problem, neither the capacity of the epidemiologist or public health planner to forecast reliably the long-term treatment needs of the $5-10 \%$ of the general population who can be expected to develop severe depressive illnesses, nor the ability of the clinician to predict the course of the condition in the individual case, has improved significantly. Trial-and-error remains the rule rather than the exception in the treatment and management of the majority of depressive episodes in most populations.

In a cross-cultural context, several studies (including the WHO multi-centre investigations) have demonstrated that, contrary to previous assumptions, depressive syndromes are common in traditional cultures and that patients in developing countries and in developed countries often present with very similar clinical features. However, little is known about the 'natural history' of depression in different cultures and it is uncertain if predictors of course and outcome derived in one culture can be applied in the assessment of patients in another.

An account of gaps and deficiencies in knowledge is already an implicit statement about priorities for future research. In regard to collaborative research of the kind that can be stimulated and facilitated by international bodies like WHO, there are at present several explicit objectives and priorities.

(1) An overriding task is the development of an agreed, 'state-of-the-art' diagnostic classification of the affective disorders which should be practically useful to clinicians, instructive to students, and appealing to research workers. Such a classification should be systematically tested for aetiological, therapeutic and prognostic implications. An opportunity to design such a classification will be the preparation of the 10th revision of the International Classification of Diseases. A provisional 
structure for the classification of affective disorders in ICD-10 is currently being designed. The development of the new classification and of a 'family' of assessment instruments and their pilot testing before release for general use is an important and urgent priority for WHO.

(2) A second objective is the design and implementation of prospective studies in which the combined effects of different classes of predictors, including biological, symptomatological, personality-related and social variables would be explored in representative samples of depressive patients in different populations, selected on epidemiological principles. The inclusion in such research of patient cohorts in different cultures would permit an exploration of the cross-cultural 'robustness' of predictors of course and outcome in depressive illness.

(3) A third objective is the investigation of the course and outcome of those forms of depressive disorders that are either implicated in the new epidemiological trends mentioned above, or are seen in large numbers in settings different from the psychiatric clinic or hospital. These are, above all, the depressions occurring in very young males and females (including adolescents and children), and the depressive illnesses seen by general practitioners, other primary care workers, or other medical specialists (e.g. internists, endocrinologists, geriatric physicians).

(4) Other objectives for collaborative research could include: (i) the improvement of the prediction of therapeutic response, with special emphasis on the need for simple and low-cost assessment procedures which do not require specialized laboratory facilities and can be applied by the primary health worker; (ii) the early identification of treatment-resistant depressions; and (iii) the refinement of the diagnostic and prognostic criteria for depressive states in the elderly.

A. JABLENSKY

\section{REFERENCES}

Akiskal, H. S., Bitar, A. H., Puzantian, V. P., Rosenthal, T. L. \& Walker, P. W. (1978). The nosological status of neurotic depression. A prospective three-to-four-year follow-up examination in light of the primary-secondary and unipolar-bipolar dichotomies. Archives of General Psychiatry 35, 756-766.

Akiskal, H. S., Hirschfeld, R. M. A. \& Yerevanan, B. I. (1983a). The relationship of personality to affective disorders. Archives of General Psychiatry 40, 801-810.

Akiskal, H. S., Walker, P., Puzantian, V. P., King D., Rosenthal, T. L. \& Dranon, M. $(1983 \mathrm{~b})$. Bipolar outcome in the course of depressive illness. Phenomenologic, familial, and pharmacologic predictors. Journal of Affective Disorders 5, 115-128.

Akiskal, H. S., Lemmi, H., Dickson H., King, D., Yerevanian, B. \& van Valkenburg, C. (1984). Chronic depressions. Part 2. Sleep EEG differentiation of primary dysthymic disorders from anxious depressions. Journal of Affective Disorders 6, 287-295.

American Psychiatric Association (1980). Diagnostic and Statistical Manual of Mental Disorders (third edn). APA: Washington, D.C.

Angst, J. (1979). Dépressions chez le sujet âgé. Résultat d'une étude longitudinale. L'Encéphale 5, 463-473.

Angst, J. (1980). Verlauf unipolar depressiver, bipolar manischdepressiven und schizo-affektiver Erkrankungen und Psychosen. Ergebnisse einer prospektiven Studie. Fortschritte der Neurologie und Psychiatrie 48, 3-30.

Angst, J., Frey, R., Felder, W. \& Lohmeyer, B. (1981). The course of monopolar depression and bipolar manic-depressive psychoses and schizoaffective psychoses. In Prospective Longitudinal Research (ed. S. A. Mednick and A. E. Baert), pp.260-262. WHO Regional Office for Europe/Oxford University Press: Oxford.

Binder, J. \& Angst, J. (1981). A prospective epidemiological study of depressive, psychosomatic and neurotic disturbances. In Prospective Longitudinal Research (ed. S. A. Mednick and A. E. Baert), pp.157-160. WHO Regional Office for Europe/Oxford University Press: Oxford.

Bland, R. C. \& Orn, H. (1982). Course and outcome in affective disorders. Canadian Journal of Psychiatry 27, 573-581.

Brockington, I. F., Helzer, J. E., Hillier, V.F. \& Francis, A. F. (1982). Definition of depression: concordance and prediction of outcome. American Journal of Psychiatry 139, 1022-1027.
Brown, G W. \& Harris, T. (1978). Social Origins of Depression. Tavistock Publications: London.

Brown, G. W. \& Prudo, R. (1981). Psychiatric disorder in a rural and an urban population: 1 . Aetiology of depression. 2 . Sensitivity to loss. Psychological Medicine 11, 581-599, 601-616.

Brugger, C. (1933). Psychiatrische Ergebnisse einer medizinischen, anthropologischen und sozıologischen Bevölkerungsuntersuchung. Zeitschrift für die gesamte Neurologie und Psychiatrie 146, 489.

Bürk, F. \& Möller, H. J. (1985). Prädiktoren für weiteres suizidales Verhalten bei nach einem Suzidversuch hospitalisierten Patienten. Fortschritte der Neurologie und Psychiatrie 53, 259-270.

Copeland, J. R. M. (1983). Psychotic and neurotic depression: discriminant function analysis and five-year outcome. Psychological Medicine 13, 373-383.

Copeland, J. R. M. (1985). Depressive illness and morbid distress. Onset and development data examined against five-year outcome. British Journal of Psychiatry 146, 297-307.

Coryell, W. \& Tsuang, M. T. (1985). Major depression with mood-congruent or mood-incongruent psychotic features: outcome after 40 years. American Journal of Psychiatry 142, 479-482.

Coryell, W. \& Winokur, G. (1982). Course and outcome. In Handbook of Affective Disorders (ed. E. S. Paykel), pp.93-106. Churchill Livingstone: Edinburgh.

Dahlberg, G. \& Stenberg, S. (1931). Eine statistische Untersuchung über die Wahrscheinlichkeit der Erkrankung an verschiedenen Psychosen. Zeitschrift für die gesamte Neurologie und Psychiatrie $133,447$.

Davidson, J. \& Robertson, E. (1985). A follow-up study of post partum illness, 1946-1978. Acta Psychiatrica Scandinavica 71, 451-457.

Dunn, G. \& Skuse, D. (1981). The natural history of depression in general practice: stochastic models. Psychological Medicine 11, 755-764.

Enzell, K. (1984). Mortality among persons with depressive symptoms and among responders and non-responders in a health check-up. Acta Psychiatrica Scandinavica 69, 89-102.

Essen-Möller, E., Larsson, H., Uddenberg, C. E. \& White, G. (1956). Individual traits and morbidity in a Swedish rural population. Acta Psychiatrica et Neurologica Suppl. 100. 
Feighner, J. P., Robins, E., Guze, S. B., Woodruff, R., Winokur, G. \& Munoz, R. (1972). Diagnostic criteria for use in psychiatric research. Archives of General Psychiatry 26, 57-63.

Feinberg, M. \& Carroll, B. J. (1984). Biological 'markers' for endogenous depression. Archives of General Psychiatry 41, 1080-1085.

Glen, A. I. M., Johnson, A. L. \& Shepherd, M. (1984). Continuation therapy with lithium and amitriptyline in unipolar depressive illness: a randomized, double-blind, controlled trial. Psychological Medicine 14, 37-50.

Greden, J. F., Price, H. L., Genero, N., Feinberg, M \& Levine, S. (1984). Facial EMG activity levels predict treatment outcome in depression. Psychiatry Research 13, 345-352.

Guze, S. B. \& Robins, E. (1970). Suicide and primary affective disorder. British Journal of Psychiatry 117, 437-438.

Hagnell, O., Lanke, J., Rorsman, B. \& Ojesjö, L. (1982). Are we entering an age of melancholy? Depressive illnesses in a prospective epidemiological study over 25 years: the Lundby Study, Sweden. Psychological Medicine 12, 279-289.

Helgason, L. (1977). Psychiatric services and mental illness in Iceland. Acta Psychiatrica Scandinavica Suppl. 268

Helgason, T. (1964). Epidemiology of mental disorders in Iceland Acta Psychiatrica Scandinavica Suppl. 173.

Jablensky, A., Sartorius, N., Gulbinat, W. \& Ernberg, G. (1981). Characteristics of depressive patients contacting psychiatric services in four cultures. Acta Psychiatrica Scandinavica 63, 367-383.

Keller, M. B., Klerman, G. L., Lavori, P. W., Coryell, W., Endicott, J. \& Taylor, J. (1984) Long-term outcome of episodes of major depression. Journal of the American Medical Association 252, 788-792.

Kendell, R. E. (1976). The classification of depressions: a review of contemporary confusion. British Journal of Psychiatry 129, 15-28.

Kendell, R. E. \& Brockington, I. F. (1980). The identification of disease entities and the relationship between schizophrenia and the affective psychoses. British Journal of Psychiatry 137, 324-331.

Klerman, G. L. (1978). Affective disorders. In The Harvard Guide to Modern Psychiatry (ed. M. Armand and M. D. Nicholi), pp. 253-281. Belknap Press: Cambridge, Mass.

Kraepelin, E. (1921). Manic-Depressive Insanity and Paranoia (transl. R. M. Barclay). Livingstone: Edinburgh.

Maas, J. W., Koslow, S. H., Katz, M. M., Bowden, C. L., Gibbons, R. L., Stokes, P. E., Robins, E. \& Davis, J. M. (1984). Pretreatment neurotransmitter metabolite levels and response to tricyclic antidepressant drugs. American Journal of Psychiatry 141, 1159-1171.

Medical Research Council. Clinical Psychiatry Committee (1965) Clinical trial of the treatment of depressive illness. British Medical Journal i, 881-886.

Myers, J. K., Weissman, M. M., Tischler, G. L., Holzer, C. E., Leaf, P. J., Orvaschel, H., Anthony, J. C., Boyd, J. H., Burke, J. D Kramer, M. \& Stolzman, R. (1984). Six-month prevalence of psychiatric disorders in three communities. Archives of General Psychiatry 41, 959-967.

Nielsen, J. (1976). The Samsö project from 1957 to 1974. Acta Psychiatrica Scandinavica 54, 198

Paykel, E. S., Prusoff, B. A. \& Tanner J. (1976). Temporal stability of symptom patterns in depression. British Journal of Psychiatry 128, 369-374.

Prien, R. F., Kupfer, D. J., Mansky, P. A., Small, J. G., Tuason, V. B., Voss, C. B. \& Johnson, W. E. (1984). Drug therapy in the prevention of recurrences in unipolar and bipolar affective disorders. Archives of General Psychiatry 41, 1096-1104.

Quitkin, F. M., Gittelman-Kleın, R., Rifkin, A, \& Klein, D. (1975). Atypical signs of relapse in affective disorders. Diseases of the Nervous System 36, 145-146.
Robins, L. N., Helzer, J. E., Weissman, M. M., Orvaschel, H., Gruenberg, E., Burke, J. D. \& Regier, D. A. (1984). Lifetime prevalence of specific psychiatric disorders in three sites. Archives of General Psychiatry 41, 949-958.

Sartorius, N. (1978). Depressive disorders, a major public health problem. In Mood Disorders: The World's Major Public Health Problem (ed. F. J. Ayd and I. J. Taylor). pp. 1-8. Ayd Medical Communications, Baltimore, $\mathrm{Md}$.

Sartorius, N., Davidian, H., Ernberg, G., Fenton, F. R, Fujii, I., Gastpar, M., Gulbinat, W., Jablensky, A, Kielholz, P., Lehmann, H. E., Naraghi, M., Shimizu, M., Shinfuku, N. \& Takahashi, R. (1983). Depressive Disorders in Different Cultures. WHO: Geneva.

Saugstad, L. F. \& Ødegaard, Ø. (1979). Mortality in psychiatric hospitals in Norway 1950-74. Acta Psychiatrica Scandinavica 59, 431-447.

Schildkraut, J. J., Orsulak, P. J., Schatzberg, A. F., Gudeman, J. E., Cole, J. O., Rohde, W. A. \& Labrie, R. A. (1978). Toward a biochemical classification of depressive disorders. Archives of General Psychiatry 35, 1427-1433.

Sheldrick, C., Jablensky, A., Sartorius, N. \& Shepherd, M. (1977). Schizophrenia succeeded by affective illness: catamnestic study and statistical enquiry. Psychological Medicine 7, 619-624.

Spitzer, R. L., Endicott, J. \& Robins, E. (1977). Research Diagnostic Criteria $(R D C)$ for a Seclected Group of Functional Disorders (3rd edn). New York State Psychıatric Institute: New York.

Strömgren, E. (1938). Beiträge zur psychiatrischen Erblehre, auf Grund von Untersuchungen an einer Inselbevölkerung. Acta Psychiatrica et Neurologica Suppl. 19.

Träskman, L., Åsberg, M., Bertilsson, L. \& Sjostrand, L. (1981). Monoamine metabolites in CSF and suicidal behaviour. Archives of General Psychiatry 38, 631-636.

Tsuang, M. T. (1978). Suicide in schizophrenics, manics, depressives, and surgical controls. Archives of General Psychiatry 35, 153-155.

Tsuang, M. T. (1980). Social effects of schizophrenia and affective disorders. In The Social Consequences of Psychiatric Illness (ed. L. N. Robins, P. J. Clayton and J. K. Wing), pp. 209-215. Brunner/ Mazel: New York.

Tsuang, M. T. \& Woolson J. R. F. (1978). Excess mortality in schizophrenia and affective disorders. Archives of General Psychiatry 35, 1181-1185.

Tsuang, M. T., Woolson, J. R. F. \& Fleming, J. A. (1980). Premature deaths in schizophrenia and affective disorders. Archives of General Psychiatry 37, 979-983.

Videbech, T. (1975). A study of genetic factors, childhood bereavement, and premorbid personality traits in patients with anancastic endogenous depression. Acta Psychiatrica Scandinavica 52, 178-222.

Weissman, M. M. (1985). Does psychiatric epidemiology count for the clinician? Paper presented at Special Lecture Series, 138th Annual Meeting of the American Psychiatric Association, Dallas, Texas, May 1985.

Wing, J. K., Cooper, J. E. \& Sartorius, N. (1974). The Measurement and Classification of Psychiatric Symptoms. Cambridge University Press: Cambridge.

Winokur, G. (1975). The Iowa 500: heterogeneity and course in manic-depressive illness (bipolar). Comprehensive Psychiatry 16, 125-131.

World Health Organization (1978). Mental Disorders: Glossary and Guide to their Classification in Accordance with the Ninth Revision of the International Classification of Diseases. WHO: Geneva.

World Health Organization (1979). Schizophrenia: An International Follow-up Study. Wiley \& Sons: Chichester. 